\title{
Comportamentos Alimentares Problemáticos após Cirurgia Bariátrica: Um Estudo com Amostra Nacional Portuguesa
}

\author{
Problematic Eating Behaviors after Bariatric Surgery: A \\ National Study with a Portuguese Sample
}

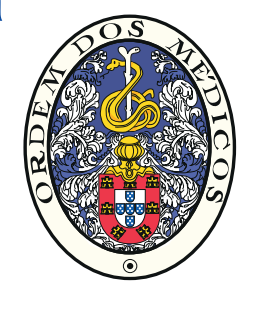

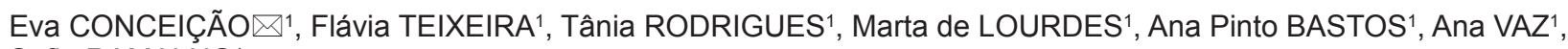 \\ Sofia RAMALHO ${ }^{1}$
}

Acta Med Port 2018 Nov;31(11):633-640 • https://doi.org/10.20344/amp.9237

\section{RESUMO}

Introdução: A cirurgia bariátrica é o método mais eficaz para o tratamento da obesidade mórbida. No entanto, o desenvolvimento de psicopatologia alimentar e o aparecimento de comportamentos alimentares problemáticos têm sido relatados na literatura. Este estudo transversal teve como objetivo a caracterização da população pós-cirurgia bariátrica em termos da frequência de comportamentos alimentares problemáticos ao longo do tempo e a compreensão das características psicológicas associadas.

Material e Métodos: A amostra foi constituída por 155 doentes sujeitos a cirurgia bariátrica que responderam a instrumentos de autorrelato destinados a avaliar psicopatologia alimentar, comportamentos alimentares problemáticos, ansiedade, depressão e stress e comportamento impulsivo.

Resultados: Os resultados mostram que o petisco contínuo, a ingestão alimentar compulsiva, a preocupação com o peso e com a forma e a urgência negativa são significativamente mais frequentes 24 meses após a cirurgia bariátrica (comparativamente com momentos pós-operatórios mais precoces). A psicopatologia alimentar e a presença de comportamentos alimentares problemáticos correlacionam-se significativa e positivamente com níveis de ansiedade, depressão, stress e urgência negativa. Este estudo reforça ainda o papel mediador da urgência negativa na relação entre tempo decorrido desde a cirurgia e distress psicológico, e comportamentos alimentares problemáticos, explicando $32,3 \%$ e $27,2 \%$ da variância, respectivamente.

Discussão: Os resultados apontam para uma tendência crescente de doentes a reportar comportamentos alimentares problemáticos e níveis de impulsividade ao longo do tempo.

Conclusão: Tendo em conta a evidência estabelecida do seu impacto na perda e aumento ponderal, a identificação precoce de comportamentos alimentares problemáticos e de doentes com tendência para agir impulsivamente em situações de emocionalidade negativa deve ser central no acompanhamento do doente sujeito a cirurgia bariátrica.

Palavras-chave: Cirurgia Bariátrica; Comportamentos Alimentar; Compulsão Alimentar/psicologia; Obesidade Mórbida/cirurgia; Petisco Contínuo

\section{ABSTRACT}

Introduction: Bariatric surgery is the most effective method for the treatment of morbid obesity. However, the development of eating psychopathology and the emergence of problematic eating behaviours have been reported in the literature. The aim of this crosssectional study was to characterize the post-bariatric population in terms of the frequency of problematic eating behaviours over time and to understand the related psychological features.

Material and Methods: This sample was composed of 155 bariatric patients that responded to several self-reported instruments assessing eating psychopathology, problematic eating behaviours, anxiety, depression and stress and impulsive behaviour.

Results: Results showed that grazing, binge eating, concerns about body weight and shape, and negative urgency are significantly more frequent at 24 months after bariatric surgery (when compared to earlier postoperative assessments). Correlational analyses showed that eating psychopathology and problematic eating behaviours were significantly and positively associated with levels of anxiety, depression, stress and negative urgency. This study also reinforces the mediating role of negative urgency in the relationship between time elapsed since surgery and psychological distress, and problematic eating behaviours, accounting for a total of $32.3 \%$ and $27.2 \%$ of its variance, respectively.

Discussion: The results suggest a growing trend of problematic eating behaviours and levels of impulsivity being reported by bariatric patients over time.

Conclusion: Given the established evidence that supports its impact on weight variability, early identification of problematic eating behaviours and of patients with a tendency to act impulsively in situations of negative emotionality should be a central concern in the follow-up of the bariatric population.

Keywords: Bariatric Surgery; Feeding and Eating Disorders/psychology; Feeding Behavior; Obesity, Morbid/surgery; Snacks

\section{INTRODUÇÃO}

A obesidade, caracterizada pela concentração atípica ou exagerada de gordura corporal, é considerada pela Organização Mundial de Saúde uma das maiores preocupações para a sociedade moderna pela elevada taxa de mortalidade e prevalência associadas. ${ }^{1}$
O tratamento considerado mais eficaz para a obesidade mórbida é a cirurgia bariátrica, que está indicada para indivíduos com um índice de massa corporal (IMC) igual ou superior a $40 \mathrm{~kg} / \mathrm{m}^{2}$, ou igual ou superior a $35 \mathrm{~kg} /$ $\mathrm{m}^{2}$ na presença de comorbilidades médicas associadas. ${ }^{2}$

1. Centro de Investigação em Psicologia. Escola de Psicologia. Universidade do Minho. Braga. Portugal.

$\triangle$ Autor correspondente: Eva Conceição. econceicao@psi.uminho.pt

Recebido: 07 de setembro de 2017 - Aceite: 02 de agosto de 2018 | Copyright @ Ordem dos Médicos 2018 
Esta cirurgia, para além de permitir uma considerável perda ponderal e a melhoria ou resolução das comorbilidades associadas a longo prazo, está também associada a meIhorias significativas a nível psicológico, físico, e social. ${ }^{3,4}$

Apesar das melhorias vivenciadas por estes doentes, os resultados do tratamento cirúrgico para a obesidade têm mostrado variabilidade significativa a longo prazo, tanto na perda de peso e sua manutenção, ${ }^{5}$ como no estado psicológico do doente sujeito a cirurgia. ${ }^{6} \mathrm{Em}$ particular, o desenvolvimento de perturbações do comportamento alimentar e o aparecimento de comportamentos alimentares problemáticos (CAPs) têm sido relatados na literatura, ${ }^{7-9}$ tornando-se esta temática alvo de preocupação para investigadores e clínicos.

Com efeito, após a cirurgia, e com vista à perda ponderal, pretende-se que o doente adira a um plano alimentar restritivo que implica a monitorização da quantidade e qualidade dos alimentos ingeridos. De acordo com Conceição et $a l,{ }^{10}$ apesar de se pretenderem temporários, estes hábitos alimentares restritivos são, em alguns casos, mantidos a longo prazo como forma de exercer controlo sobre o peso, através da implementação de regras alimentares rígidas. Deste modo, o alerta recorrente da possibilidade de aumento de peso pelos profissionais de saúde, a diminuição da taxa de perda de peso, ou as variações de peso normais a que se assiste quando se atinge o plateau na perda de peso parecem desencadear preocupações significativas com o controlo do peso e com a alimentação num subgrupo de doentes, podendo vir a facilitar o desenvolvimento de perturbações do comportamento alimentar após a cirurgia bariátrica. ${ }^{10}$ De facto, alguns doentes bariátricos relatam recorrer a estratégias comportamentais desadequadas de controlo de peso, ${ }^{9}$ pelo que é necessária uma atenção acrescida na avaliação destas perturbações psiquiátricas e dos seus sintomas.

Também a presença de CAPs no momento pós-operatório, e não no pré-operatório, tem vindo a ser indicada na literatura como fator de risco para piores resultados de perda de peso. ${ }^{8-10}$ Além das perturbações alimentares, diferentes CAPs, que não preenchem critérios de diagnóstico pelo DSM-5, ${ }^{11}$ têm vindo a ser identificados. A presença de petisco contínuo, ${ }^{12}$ de perda de controlo sobre a alimentação (independentemente das quantidades ingeridas) e episódios de ingestão alimentar compulsiva, ${ }^{13}$ ou a ingestão emocional, ${ }^{14}$ em particular após 1,5 - 2 anos da cirurgia, parece associar-se a menor perda de peso e/ou aumento de peso. ${ }^{8}$

Assim, este estudo tem como principal objetivo a caracterização da população pós-cirurgia bariátrica em contexto comunitário, em termos dos CAPs e características psicológicas numa amostra portuguesa, procurando compreender a relação entre o tempo decorrido após a cirurgia bariátrica, os estados psicossociais e os CAPs.

\section{MATERIAL E MÉTODOS}

\section{Amostra e procedimento}

Este é um estudo transversal desenhado para avaliar a frequência de comportamentos alimentares problemáticos após a intervenção cirúrgica e compreender as características psicológicas associadas. Os participantes foram recrutados, em colaboração com a Associação Portuguesa dos Bariátricos (APOBARI), por divulgação online em seis grupos sociais de doentes bariátricos na rede social Facebook $^{\circledR}$, onde apenas os membros dos grupos têm acesso à informação publicada. São membros da APOBARI e integram estes grupos doentes de todo o território nacional. Constituíram critérios de inclusão: ter mais de 18 anos; ter sido submetido a cirurgia bariátrica; ser membro de, pelo menos, um dos grupos sociais de Facebook. Como critérios de exclusão foram consideradas a gravidez ou lactação.

A recolha de dados foi totalmente realizada online. Durante 20 semanas, entre novembro de 2016 e março de 2017, e com frequência quinzenal, a equipa de investigação publicou um post, convidando à participação no estudo, que incluía uma hiperligação para o preenchimento da bateria de testes na aplicação Google Drive. Numa primeira fase foi descrito o estudo e os seus objetivos, sendo realçado o caráter voluntário e anónimo da participação no estudo. O consentimento informado foi considerado na aceitação das condições do estudo. Foi permitida uma participação por pessoa.

\section{Instrumentos}

\section{Questionário sociodemográfico e clínico}

Este questionário foi utilizado para recolher informação sobre a idade, estado civil, nível de escolaridade, situação profissional, tipo de cirurgia bariátrica realizada, tempo decorrido desde a cirurgia, e recurso a suplementos nutricionais.

\section{Eating disorder-15 (ED-15) ${ }^{15}$}

Traduzido por Machado (2016). O ED-15 é utilizado para avaliar sintomas de perturbações alimentares, sendo constituído por quinze itens. Dez avaliam atitudes associadas à psicopatologia do comportamento alimentar e são respondidos numa escala tipo Likert de sete pontos ( $0=$ Nada; a 6 = Sempre), gerando duas subescalas (preocupação com a comida e preocupação com o peso e forma corporal). Para este estudo foram usados os quatro itens que avaliam o uso de métodos compensatórios.

\section{Escala de ansiedade, depressão e stress-21 (EADS-} 21) $)^{16,17}$

A escala é constituída por vinte e uma questões, distribuídas por três subescalas (ansiedade, depressão e stress), sendo respondidas tendo em conta a última semana, numa escala tipo Likert de quatro pontos (0 = Não se aplicou nada a mim; a 3 = Aplicou-se a mim a maior parte das vezes). Esta escala é utilizada para avaliar estados afetivos, onde pontuações mais elevadas revelam estados afetivos mais negativos. Para este estudo foi usada a soma das três subscalas (EADS-21_Total) como medida de distress psicológico. 


\section{Escala do comportamento impulsivo (UPPS-P) ${ }^{18}$}

A UPPS-P é constituída por cinquenta e nove itens distribuídos por cinco subescalas (urgência negativa, falta de premeditação, falta de perseverança, procura de sensações e urgência positiva). Esta escala permite avaliar a impulsividade com base nas dimensões do Modelo dos Cinco Fatores da Personalidade. Neste estudo, apenas foi administrada a subescala 'Urgência negativa', que indica a tendência apara agir de forma impulsiva em situações de emocionalidade negativa. É composta por doze itens, numa escala tipo Likert de quatro pontos $(1=$ Concordo completamente; a 4 = Discordo completamente).

\section{Questionário de avaliação de comportamentos ali- mentares problemáticos (QACAP)}

Desenvolvido pelos autores para este estudo, este instrumento questiona o número de dias, durante a última semana, em que ocorreram os seguintes CAPs: i) saltar refeições; ii) petisco contínuo ('petiscou ou debicou pequenas quantidades de comida de uma forma não planeada e repetida ao longo do dia'; iii) ingestão excessiva ('comeu em exagero ou mais do que devia ter comido'); iv) ingestão emocional ('comeu em exagero ou não resistiu comer por se sentir muito ansioso/a, nervoso/a, triste ou por outras emoções'); v) perda de controlo sobre a alimentação ('sentiu que perdeu o controlo sobre o que estava a comer'); vi) episódios de ingestão alimentar compulsiva ('sentiu que comeu compulsivamente, comendo descontroladamente quantidades exageradamente grandes de comida para as circunstâncias').

\section{Análise de dados}

Os dados recolhidos foram sujeitos a tratamento e análise estatística através do programa Statistical Package for Social Sciences ${ }^{\circledR}$ (SPSS) - versão 24 (para Windows).

Neste estudo foram realizadas análises de estatística descritiva e $\chi^{2}$ para caracterizar a amostra e determinar a percentagem de participantes que reportaram cada um dos comportamentos alimentares avaliados em diferentes momentos temporais. A análise unifatorial de variância (ANOVA) e a análise multifatorial de variância (MANOVA) foram usadas para investigar diferenças entre os períodos de tempo decorridos desde a cirurgia bariátrica nas variáveis psicológicas avaliadas. Correlações de Spearman testaram a correlação existente entre as variáveis psicológicas avaliadas e a presença de comportamentos alimentares problemáticos. Por fim, apesar de este ser um estudo transversal, foram testados dois modelos de mediação que nos permitiram estudar a percentagem de variância explicada da relação entre duas variáveis (ex: tempo decorrido desde a cirurgia ou distress psicológico e CAPs) através do efeito mediador de uma terceira variável (ex: urgência negativa). O macro PROCESS para o SPSS foi usado com bootstrapping de 5000 e $95 \%$ de intervalo de confiança. Valores de $p$ $<0,05$ foram considerados estatisticamente significativos.

\section{RESULTADOS}

A amostra foi constituída por 155 participantes, 143 $(92,3 \%)$ do sexo feminino e $12(7,7 \%)$ do sexo masculino. A idade dos participantes variou entre os 24 e os 62 anos, sendo a média de idades de 40,37 ( $D P=8,1)$. No que se refere ao nível de escolaridade, $9 \%$ dos participantes concluíram o ensino básico, $44,5 \%$ completaram o ensino secundário e $46,5 \%$ completaram o ensino superior.

Relativamente ao estado civil, a maioria dos participantes $(61,3 \%)$ eram casados ou viviam com o/a companheiro/a, $31 \%$ eram solteiros e $7,7 \%$ eram divorciados. No que diz respeito à situação profissional, $81,3 \%$ encontravam-se empregados, enquanto $16,1 \%$ estavam desempregados, e $2,6 \%$ eram estudantes. A maioria dos participantes foi submetida a bypass gástrico $(69,7 \%), 26,5 \%$ a sleeve gástrico e $3,8 \%$ a outro tipo de cirurgia bariátrica ou desconhecem qual o procedimento cirúrgico realizado. Relativamente ao tempo de pós-operatório, 47 participantes (30,3\%) tinham menos de seis meses; $34(21,9 \%)$ entre seis e 11 meses; $28(18,1 \%)$ entre 12 e 23 meses; e $46(29,7 \%) 24$ meses ou

Tabela 1 - Percentagem e número médio de dias, na última semana ou último mês, em que os participantes reportaram padrões alimentares disfuncionais ao longo dos períodos de tempo decorridos desde a cirurgia bariátrica, avaliados através do QACAP e ED-15

\begin{tabular}{|c|c|c|c|c|c|c|}
\hline & $\begin{array}{c}0-5 \text { meses } \\
n(\%)\end{array}$ & $\begin{array}{c}6-11 \text { meses } \\
n(\%)\end{array}$ & $\begin{array}{c}12-23 \text { meses } \\
\mathrm{n}(\%)\end{array}$ & $\begin{array}{c}\geq 24 \text { meses } \\
n(\%)\end{array}$ & $\begin{array}{l}\text { Total } \\
\mathrm{n}(\%)\end{array}$ & $\chi^{2}(3)$ \\
\hline QACAP - Petisco contínuo & $21(44,7)$ & $28(82,4)$ & $24(85,7)$ & $41(89,1)$ & $114(73,5)$ & $29,36^{*}$ \\
\hline QACAP - Saltar refeições & $31(66,0)$ & $13(38,2)$ & $13(46,4)$ & $28(60,9)$ & $85(54,8)$ & $7,61^{\S}$ \\
\hline QACAP - Ingestão excessiva & $16(34,0)$ & $21(61,8)$ & $14(50,0)$ & $34(73,9)$ & $85(54,8)$ & $15,89^{*}$ \\
\hline QACAP - Ingestão emocional & $14(29,8)$ & $17(50,0)$ & $14(50,0)$ & $33(71,7)$ & $78(50,3)$ & $16,37^{*}$ \\
\hline $\begin{array}{l}\text { QACAP - Perda de controlo } \\
\text { sobre a alimentação }\end{array}$ & $13(27,7)$ & $17(50,0)$ & $12(42,9)$ & $30(65,2)$ & $72(46,5)$ & $13,50^{*}$ \\
\hline $\begin{array}{l}\text { QACAP - Ingestão alimentar } \\
\text { compulsiva }\end{array}$ & $8(17,0)$ & $5(14,7)$ & $8(28,6)$ & $24(52,2)$ & $45(29,0)$ & $18,64^{*}$ \\
\hline ED-15 - Vómito & $8(17,0)$ & $1(2,9)$ & $3(10,7)$ & $6(13,0)$ & $18(11,6)$ & 3,94 \\
\hline ED-15 - Uso de laxantes & $5(10,6)$ & n.o. & $2(7,1)$ & $5(10,9)$ & $12(7,7)$ & 4,05 \\
\hline ED-15 - Dieta restritiva & $31(66,0)$ & $20(58,8)$ & $13(46,4)$ & $28(60,9)$ & $92(59,4)$ & 2,84 \\
\hline $\begin{array}{l}\text { ED-15 - Exercício físico } \\
\text { excessivo }\end{array}$ & $16(34,0)$ & $13(38,2)$ & $16(57,1)$ & $18(39,1)$ & $63(40,6)$ & 4,13 \\
\hline
\end{tabular}

ED-15: Eating disorder-15; QACAP: Questionário de avaliação de comportamentos alimentares problemáticos; n.o.: não observado; * $p<0,01 ;{ }^{\S} p<0,1$ 
mais. De ressaltar que a maioria dos participantes $(60,6 \%)$ reportou recorrer a suplementação nutricional diariamente.

A Tabela 1 apresenta a percentagem de participantes, de cada momento pós-operatório, que referiu, na semana anterior, pelo menos um dos CAPs avaliados. De acordo com os resultados, todos os CAPs parecem ser estatisticamente diferentes entre os grupos de diferentes períodos de tempo decorridos desde a cirurgia, com exceção do comportamento de 'saltar refeições' que se mostrou marginalmente significativo. Ainda assim, observamos uma importante percentagem $(60 \%-66 \%)$ de participantes a reportar saltar refeições nos grupos com menos de seis meses e mais de 24 meses de cirurgia. O petisco contínuo é referido por uma percentagem bastante elevada de participantes (> $82 \%$ ) a partir dos seis meses de cirurgia. $O$ grupo com mais de 24 meses apresenta ainda uma maior percentagem de participantes (> 50\%) a reportar com maior frequência ingestão excessiva, ingestão emocional, perda de controlo sobre a alimentação e episódios de ingestão alimentar compulsiva. Relativamente aos comportamentos compensatórios avaliados pelo ED-15, não foram encontradas diferenças significativas entre os grupos com diferentes tempos decorridos desde a cirurgia.

A Tabela 2 descreve, para cada momento pós-operatório, os resultados obtidos nas dimensões psicológicas avaliadas pelos restantes questionários de autorrelato: preocupação com peso e forma corporal e preocupação com a comida (ED-15); ansiedade, depressão e stress (EADS21); e urgência negativa (UPPS-P).

Numa primeira observação, é o grupo com mais de 24 meses de cirurgia que apresenta valores mais elevados nas diferentes medidas de autorrelato. No entanto, os resultados espelham diferenças modestas que nem sempre atingem significância estatística.

No que respeita aos resultados do ED-15, não se verificaram diferenças estatisticamente significativas univariadas, em função dos períodos de tempo decorridos desde a cirurgia, ao nível da subescala preocupação com a comida. Contudo, ao nível da subescala preocupação com o peso e forma corporal, foram encontradas diferenças multivariadas estatisticamente significativas, com os testes post-hoc
LSD sugerindo que os doentes com menos de seis meses e mais de 24 meses após a cirurgia apresentam valores significativamente mais elevados de preocupação com a forma corporal.

Considerando as subescalas do EADS-21, não foram encontradas diferenças estatisticamente significativas univariadas em função dos períodos de tempo decorridos desde a cirurgia bariátrica ao nível das subescalas de ansiedade, depressão, e stress.

Finalmente, foram encontradas diferenças estatisticamente significativas ao nível da urgência negativa entre os períodos de tempo decorridos desde a cirurgia bariátrica. O teste post-hoc LSD revelou que os doentes pós-cirurgia, com 0 a 5 meses desde a cirurgia, revelam menor urgência negativa do que doentes com outros tempos decorridos desde a cirurgia.

Apesar de terem sido encontradas poucas diferenças significativas entre os grupos com diferentes tempos desde a cirurgia relativamente às variáveis psicológicas, estas foram encontradas nas variáveis comportamentais avaliadas pelo QACPA. No sentido de compreender que variáveis se associam aos problemas do comportamento alimentar, foram analisadas as correlações entre as variáveis em estudo (Tabela 3). Para esta análise foi considerada a variável 'Comportamentos alimentares problemáticos' (CAPs) que resulta da soma das variáveis que avaliam petisco contínuo, ingestão excessiva, ingestão emocional, perda de controlo sobre a alimentação e episódios de ingestão alimentar compulsiva. Deste modo, a variável CAPs indica o número de dias na última semana com ocorrência de pelo menos um dos CAPs avaliados. Os testes de Spearman mostraram que quanto maior o tempo decorrido desde a cirurgia, maiores os níveis de urgência negativa e mais frequentes os CAPs. Podemos também observar que a presença de CAPs está significativa e positivamente correlacionada com maiores níveis de ansiedade, depressão, stress e de urgência negativa. Por fim, maior psicopatologia relacionada com as perturbações do comportamento alimentar (avaliada pelo ED-15), e em particular a preocupação com o peso e forma corporal, encontra-se também significativa e positivamente correlacionada com maiores

Tabela 2 - Diferenças nas subescalas do ED-15, EADS-21 e UPPS em função dos períodos de tempo decorridos desde a cirurgia bariátrica

\begin{tabular}{|c|c|c|c|c|c|}
\hline & $\begin{array}{c}0 \text { - } 5 \text { meses } \\
(n=47) \\
\text { Média (DP) }\end{array}$ & $\begin{array}{c}6 \text { - } 11 \text { meses } \\
(n=34) \\
\text { Média (DP) }\end{array}$ & $\begin{array}{c}12-23 \text { meses } \\
(n=28) \\
\text { Média (DP) }\end{array}$ & $\begin{array}{c}\geq 24 \text { meses } \\
(n=46) \\
\text { Média (DP) }\end{array}$ & $F(3,15)$ \\
\hline ED-15_total & $2,97(1,39)$ & $2.26(1,20)$ & $2,39(1,08)$ & $2.76(1,43)$ & 2,37 \\
\hline Preocupação com a comida & $3,24(1,45)$ & $2,72(1,15)$ & $2,92(1,40)$ & $2,95(1,47)$ & 0,97 \\
\hline Preocupação com o peso e forma & $2,70(1,83)$ & $1,81(1,60)$ & $1,87(1,44)$ & $2,57(1,78)$ & $2,79^{*}$ \\
\hline EADS-21_total & $10,96(9,71)$ & $11,62(11,16)$ & $11,57(9,39)$ & $14,17(10,90)$ & 0,85 \\
\hline Ansiedade & $3,23(2,88)$ & $2,82(2,72)$ & $3,25(3,33)$ & $4,02(3,34)$ & 1,09 \\
\hline Depressão & $2,72(3,89)$ & $3,09(4,80)$ & $2,82(3,47)$ & $4,17(4,64)$ & 1,07 \\
\hline Stress & $5,00(4,26)$ & $5,71(4,92)$ & $5,50(3,84)$ & $5,98(4,09)$ & 0,43 \\
\hline UPPS_Urg_Neg & $23,45(8,36)$ & $27,29(7,84)$ & $28,11(8,18)$ & $28,96(9,20)$ & $3,70^{* *}$ \\
\hline
\end{tabular}

ED-15: Eating disorder-15; EADS-21: Escala de ansiedade, depressão e stress-21; UPPS_Urg_Neg: Subescala urgência negativa; * $p<0,05 ;{ }^{* *} p<0,01$ 


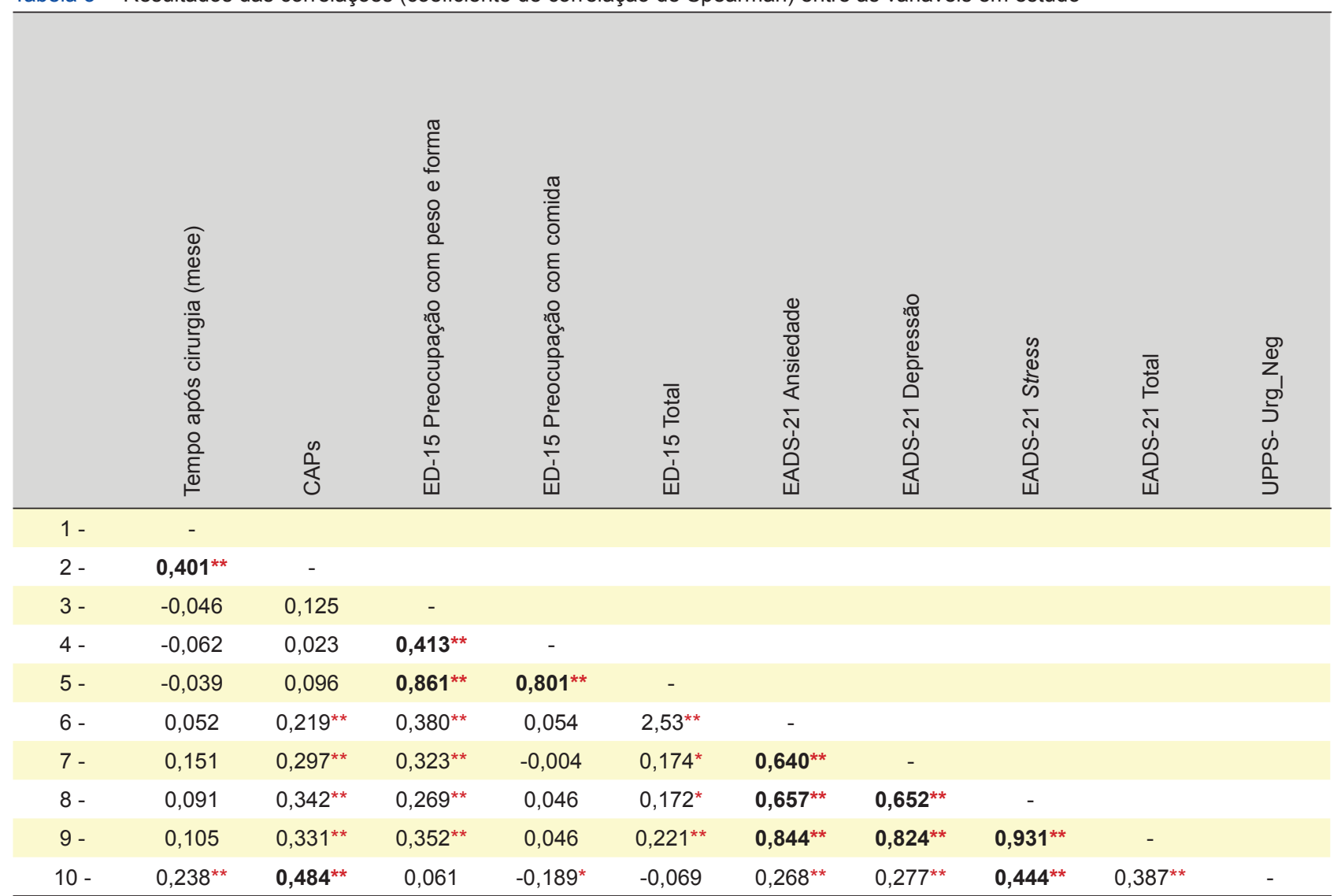

CAPs: comportamentos alimentares problemáticos; ED-15: Eating disorder-15; EADS-21: Escala de ansiedade, depressão e stress-21; UPPS_Urg_Neg: Subescala urgência negativa; valores a negrito indicam valores de correlação superiores a 0,$4 ;{ }^{*} p<0,05 ;{ }^{* *} p<0,01$

níveis de ansiedade, depressão e stress. De notar, correlações particularmente fortes $(>0,4)$ foram encontradas entre o tempo decorrido desde a cirurgia e CAPs, entre CAPs e urgência negativa, e entre urgência negativa e stress.

Tendo como um dos objetivos principais no nosso estudo compreender a ocorrência de CAPs ao longo do tempo nesta população, foram testados dois modelos de mediação. O primeiro modelo foi testado com o objetivo de compreender se a ocorrência de CAPs a longo prazo, descrita na literatura como estando associada a piores resultados de perda de peso, seria melhor explicada por uma tendência para ações impulsivas perante situações de emocionalidade negativa (urgência negativa). A confirmar a nossa hipótese, os resultados mostram que a urgência negativa nos ajuda a explicar a relação entre o tempo decorrido desde a cirurgia e a presença de CAPs.

As análises de mediação revelaram que a urgência negativa desempenha um papel mediador na relação entre o tempo decorrido desde a cirurgia e os CAPs, F (2152) = $36,28, p<0,001$, sendo que este modelo explica $32,3 \%$ do total da variância dos CAPs. Foi ainda verificado um efeito indireto significativo, $b=0,026, \mathrm{Bca} \mathrm{Cl}[0,005 ; 0,050]$, e um efeito direto significativo ( $b=0,061, p=0,001)$ - (Fig. 1).

Os resultados deste modelo de mediação sugerem que,

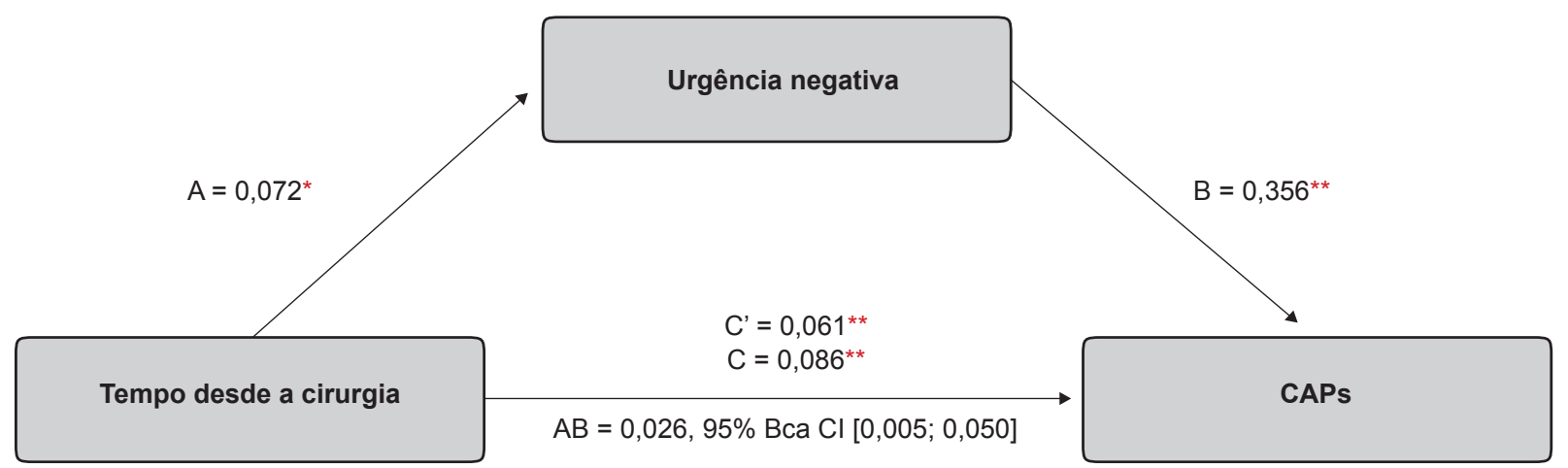

Figura 1 - Valores dos coeficientes do modelo de mediação: o efeito mediador da urgência negativa na relação entre o tempo decorrido desde a cirurgia e a frequência de comportamentos alimentares problemáticos (CAPs). ${ }^{*} p \leq 0,05 .{ }^{* *} p \leq 0,001$ 
quanto mais tempo decorrido desde a cirurgia, maior a tendência para a adoção de CAPs, em parte porque se assiste também a uma maior tendência para agir de modo impulsivo sobre estados emocionais negativos (quando introduzida a variável mediadora no modelo - urgência negativa - a força da relação entre a variável independente (tempo decorrido desde a cirurgia) e a variável dependente (CAPs) é reduzida $\left(C=0,086, p<0,001 ; C^{\prime}=0,061, p=0,001\right)$. Além disso, o caminho indireto (o efeito de mediação) explica cerca de $30 \%$ do efeito total (rácio do efeito indireto no efeito total $=29,6 \%$ ).

Dada a correlação significativa (Tabela 2) também encontrada entre urgência negativa e distress psicológico, e considerando a literatura existente que associa comprometimento psicológico e CAPs com piores resultados de perda de peso, ${ }^{8,19}$ hipotetizámos que uma tendência impulsiva para agir em situações de emocionalidade negativa seria um mediador da relação entre distress psicológico e CAPs. Os resultados do segundo modelo de mediação testado revelaram que a relação que existe entre distress psicológico (EADS-21_Total) e CPAs é totalmente mediada pela urgência negativa (agir de modo impulsivo em situações de emocionalidade negativa), $F(2152)=28,32, p<0,001$, sendo que este modelo explica $27,15 \%$ do total da variância dos CAPs. Foi ainda verificado um efeito indireto significativo, $b=0,133$, Bca Cl [0,079;0,207], e um efeito direto (c') não significativo ( $b=0,048, p=0,333)$ - (Fig. 2).

Os resultados deste modelo de mediação sugerem que mais distress psicológico está associado a maior frequência de CAPs, porque se associa a maiores níveis de urgência negativa (quando introduzida a variável mediadora no modelo - urgência negativa - a significância da relação entre distress psicológico e CAPs desaparece $(C=0,181 ; p$ $\left.<0,001 ; C^{\prime}=0,048, p=0,333\right)$. Além disso, o caminho indireto (o efeito de mediação) explica cerca de $74 \%$ do efeito total (rácio do efeito indireto no efeito total $=73,5 \%$ ).

\section{DISCUSSÃO}

O presente estudo pretendeu caracterizar a população pós-cirurgia bariátrica em relação a comportamentos alimentares problemáticos e psicopatologia associada. A compreensão dos problemas alimentares nesta população assume importância central pela sua associação a piores resultados de tratamento e maior comprometimento psicológico. Além disso, este estudo salienta a relação entre o tempo decorrido após a cirurgia bariátrica, os estados psicológicos e os comportamentos alimentares.

Os resultados deste estudo apontam para uma percentagem preocupante de indivíduos que se submeteram a cirurgia barátrica a reportar comportamentos alimentares problemáticos, o que tem vindo a ser associado a piores resultados de perda de peso. Cerca de $54 \%$ dos participantes reportaram saltar refeições; $73,5 \%$ petisco contínuo; $54,8 \%$ ingestão excessiva; $50,3 \%$ ingestão emocional; $46,5 \%$ compulsão alimentar e $29,0 \%$ episódios de ingestão alimentar compulsiva, pelo menos uma vez no último mês. Uma maior percentagem de participantes do grupo com mais de 24 meses após a cirurgia referiram os diferentes CPAs, o que vai ao encontro da literatura existente que argumenta que CAPs tendem a (re)aparecer após os dois anos de cirurgia. ${ }^{20}$ As percentagens encontradas no nosso estudo são superiores aos valores reportados na literatura, ${ }^{21}$ o que poderá ser justificado pelo formato de avaliação usado neste estudo (autorrelato) e pela frequência considerada para identificar como presente um comportamento (uma vez na última semana). Não obstante, aceder à perceção do doente sobre o seu comportamento alimentar deverá ser uma estratégia central para a necessária deteção precoce destes comportamentos. Por outro lado, uma vez que a frequência destes comportamentos tende a incrementar-se ao longo do tempo, torna-se central a sua deteção precoce quando ainda não surtem impacto nos resultados de peso da cirurgia. ${ }^{8}$

Relativamente aos itens do ED-15 que avaliam a presença de comportamentos compensatórios como o vómito, uso de laxantes/diuréticos, exercício físico excessivo e restrição alimentar com o intuito de controlar o peso e a forma corporal, a distinção entre o recurso a estes comportamentos no contexto de psicopatologia ou no contexto de um tratamento cirúrgico para perda de peso tem vindo a ser descrita como desafiante para os profissionais de saúde. ${ }^{9}$ Estes comportamentos são, habitualmente, reportados por doentes com perturbações do comportamento alimentar, como métodos desadaptativos de controlo do peso

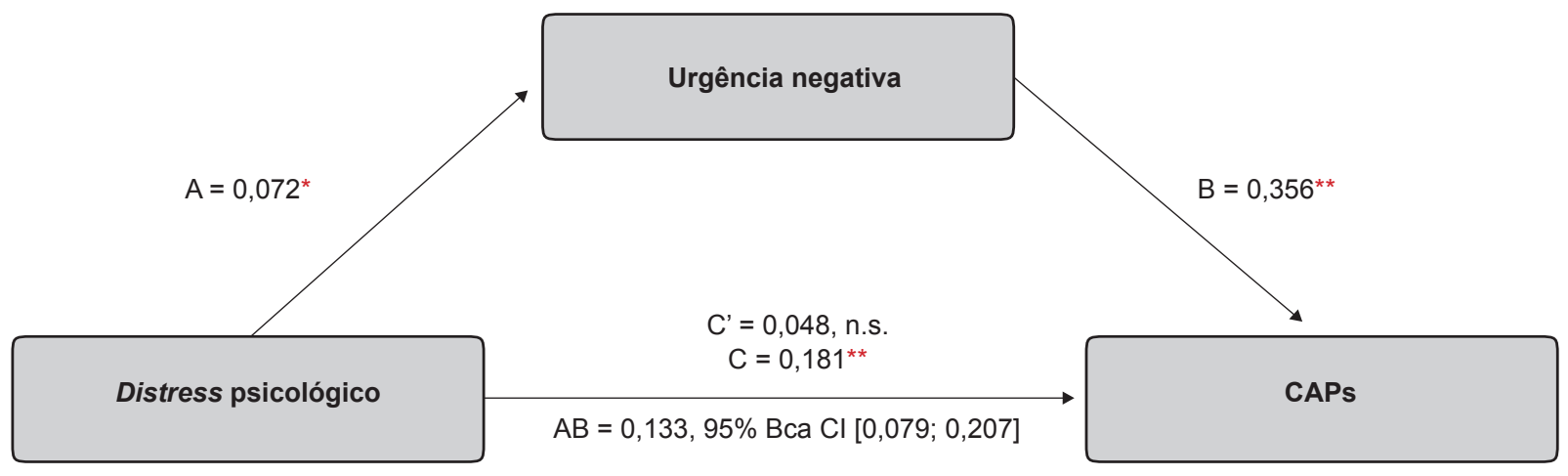

Figura 2 - Valores dos coeficientes do modelo de mediação: o efeito mediador da urgência negativa na relação entre distress psicológico a frequência de comportamentos alimentares problemáticos (CAPs).

${ }^{*} p \leq 0,05 .{ }^{* *} p \leq 0,001$ 
corporal. No entanto, no período pós-operatório, é comum a ocorrência de vómito, como reação à intolerância alimentar, e do uso de laxantes/diuréticos como terapêutica para atividade intestinal. Além disso, a prática de exercício físico e a restrição e controlo alimentar acentuados são recomendadas ao doente sujeito a cirurgia bariátrica pelos próprios profissionais de saúde. Deste modo, e tendo em conta que os nossos resultados foram obtidos por autorrelato, estes podem estar enviesados pela falta de conhecimento do significado clínico destes comportamentos. Dado que o desenvolvimento de perturbações do comportamento alimentar tem vindo a ser descrito na literatura, ${ }^{9}$ o profissional de saúde deve, portanto, estar treinado para fazer uma correta avaliação destes comportamentos.

Apesar dos resultados não mostrarem diferenças significativas na maior parte das variáveis psicológicas avaliadas (com exceção da urgência negativa), é de salientar que os resultados multivariados (mas não testes de post-hoc) sugerem diferenças entre os grupos na preocupação com o peso e forma corporal, sendo que participantes com menos de seis e mais de 24 meses após a cirurgia apresentam valores superiores (Tabela 3). Este resultado, pode ser indicador da dramática mudança no peso que o doente sujeito a cirurgia bariátrica experimenta nos primeiros seis meses e que se pode deteriorar dois anos após a cirurgia devido à existência de peles em excesso. ${ }^{22}$ No que respeita aos níveis de ansiedade, depressão e stress, a literatura existente $^{6}$ aponta para um aumento desta sintomatologia em doentes com mais de dois anos de cirurgia. Nos nossos resultados, apesar de se observarem scores superiores no grupo com mais de 24 meses, estas diferenças não se mostraram significativas. Finalmente, foram encontradas diferenças estatisticamente significativas relativamente à urgência negativa, em função dos períodos de tempo decorridos desde a cirúrgica bariátrica. Os testes de post-hoc revelaram que doentes com menos de seis meses da cirurgia apresentam valores inferiores de urgência negativa quando comparados com doentes com 24 meses ou mais de cirurgia (diferença média $=5,51 ; 95 \%$ IC $-10,20--0,82$ ). A urgência negativa, por sua vez, mostrou-se significativamente correlacionada com a presença de CAPs (Tabela 3). Deste modo, ainda que o distress psicológico (ansiedade, depressão e stress) pareça não variar de forma significativa com o tempo, participantes com mais tempo decorrido desde a cirurgia tendem a apresentar uma forma mais impulsiva de lidar com situações emocionalmente negativas, o que, por sua vez, explicava a maior presença de CAPs ao longo do tempo. Além disso, hipotetizou-se ainda que a correlação encontrada entre sintomatologia depressiva e CAPs fosse, também ela, melhor explicada pela urgência negativa. Os modelos de mediação testados sugerem que a urgência negativa é um mediador significativo da relação entre o tempo decorrido desde a cirurgia e CAPs, e entre distress psicológico e CAPs. Estes resultados contrapõem-se com os publicados por Schag et al, ${ }^{23}$ no qual se defende a possibilidade de existir um resultado indireto da impulsividade na perda de peso, possivelmente mediado através da sintomatologia depressiva e do comportamento alimentar disfuncional. No nosso estudo, porém, os modelos testados com a variável de distress psicológico no papel de mediadora (dados não apresentados) não se mostraram significativos. Estudos futuros devem explorar o papel que a urgência negativa poderá ter nos resultados de perda de peso e explorar os mecanismos através dos quais é operada tal relação.

As limitações deste estudo incluem o pequeno tamanho da amostra (155 participantes) já que os participantes foram agrupados em quatro grupos diferentes, a assimetria na proporção de homens/mulheres, e o facto da avaliação dos CAPs ter sido feita por autorrelato, o que tende a sobre-reportar a presença destes comportamentos. Não obstante, o facto dos participantes do estudo terem sido recrutados online e fora do contexto hospitalar permitiu o acesso à experiência subjetiva do participante sem o viés da influência que o profissional de saúde possa infligir no contexto de tratamento. Adicionalmente, a nossa amostra está constituída por pessoas que receberam diferentes tipos de cirurgia. Lamentavelmente, o número de pessoas que receberam outro procedimento que não o bypass gástrico era pequeno limitando a possibilidade de testar diferenças entre diferentes grupos. Estudos futuros devem explorar se estas relações ou se a frequência dos diferentes CAPs se mantém para doentes recebendo diferentes procedimentos cirúrgicos.

\section{CONCLUSÃO}

Os nossos resultados salientam a elevada frequência com que o doente sujeito a cirurgia bariátrica reporta CAPs no momento pós-operatório, assim como ao papel da tendência para agir de forma impulsiva quando sob emocionalidade negativa para explicar a presença de CPAs. Doentes com mais tempo decorrido desde a cirurgia, ou que reportem valores de distress psicológico mais elevados, tendem a agir de forma mais impulsiva em situações emocionalmente ativadoras, o que se associa a mais frequência de CAPs. Os nosso dados apontam para a importância de uma avaliação cuidada e sistemática ao longo do tempo, não só da presencia de CAPs, mas também ficada na forma como o doente lida com situações de emocionalidade negativa. Tendo em conta a evidência estabelecida do impacto de CAPs na perda e aumento de peso, ${ }^{20-21,24}$ a identificação precoce de CAPs, e o treino de competências de gestão emocional, em particular no longo prazo após a cirurgia, parecem estratégias relevantes para uma melhor adesão à alteração comportamental necessária para manter a perda de peso a longo-prazo.

\section{AGRADECIMENTOS}

Os autores desejam agradecer à APOBARI o seu apoio no desenvolvimento e divulgação deste estudo.

\section{PROTEÇÃO DE PESSOAS E ANIMAIS}

Os autores declaram que os procedimentos seguidos estavam de acordo com os regulamentos estabelecidos 
pelos responsáveis da Comissão de Investigação Clínica e Ética e de acordo com a Declaração de Helsínquia da Associação Médica Mundial.

\section{CONFIDENCIALIDADE DOS DADOS}

Os autores declaram ter seguido os protocolos do seu centro de trabalho acerca da publicação de dados.

\section{CONFLITOS DE CONFLITOS DE INTERESSE}

Os autores declaram não ter conflitos de interesse.

\section{FONTES DE FINANCIAMENTO}

Este estudo foi suportado pela Fundação para a Ciência e a Tecnologia, um programa de financiamento da União Europeia COMPETE a Eva Conceição (IF/01219/2014) e bolsa de doutoramento (SFRH/BD/104159/2014) a Ana Pinto-Bastos, (SFRH/BD/104182/2014), Sofia Ramalho e (SFRH/BPD/94490/2013), Ana Vaz. Este trabalho foi conduzido no Centro de Investigação em Psicologia (UID/ PSI/01662/2013), Universidade do Minho e parcialmente apoiado pela Fundação para a Ciência e a Tecnologia e pelo Ministério da Ciência, Tecnologia e Ensino Superior através de fundos nacionais, tendo sido ainda co-financiado pelo FEDER através do COMPETE2020 sob o PT2020 Compromisso para a Ciência (POCl-01-0145-FEDER-007653).

\section{REFERÊNCIAS}

1. World Health Organization. c2017-08. [consultado 2018 nov 20]. Disponível em: http://www.who.int/mediacentre/factsheets/fs311/en/.

2. Buchwald H. 2004 ASMBS Consensus Conference: Consensus statement Bariatric surgery for morbid obesity: health implications for patients, health professionals, and third party payers. Surg Obes Relat Dis. 2005;1:371-81.

3. Buchwald H, Avidor Y, Braunwald E, Jensen MD, Pories W, Fahrbach K, et al. Bariatric surgery. A systematic review and meta-analysis. JAMA. 2004;292:1724-37.

4. Thomson L, Sheehan KA, Meaney C, Wnuk S, Hawa R, Sockalingam S. Prospective study of psychiatric illness as a predictor of weight loss and health related quality of life one year after bariatric surgery. J Psychosom Res. 2016;86:7-12.

5. Courcoulas AP, Christian NJ, Belle SH, Berk PD, Flum DR, Garcia L, et al. Weight change and health outcomes at 3 years after bariatric surgery among individuals with severe obesity. JAMA. 2013;310:2416-25.

6. Mitchell JE, King WC, Chen JY, Devlin MJ, Flum D, Garcia L, et al. Course of depressive symptoms and treatment in the longitudinal assessment of bariatric surgery (LABS-2) study. Obesity. 2014;22:1799806.

7. Marino JM, Ertelt TW, Lancaster K, Steffen K, Peterson L, de Zwaan $M$, et al. The emergence of eating pathology after bariatric surgery: a rare outcome with important clinical implications. Int J Eat Disord. 2012;45:179-84.

8. Conceição EM, Utzinger LM, Pisetsky EM. Eating disorders and problematic eating behaviours before and after bariatric surgery: characterization, assessment and association with treatment outcomes. Eur Eat Disord Rev. 2015;23:417-25.

9. Conceição E, Orcutt M, Mitchell J, Engel S, LaHaise K, Jorgensen M, et al. Eating disorders after bariatric surgery: a case series. Int $\mathrm{J}$ Eat Disord. 2013;46:274-9.

10. Conceição E, Vaz A, Bastos AP, Ramos A, Machado P. The development of eating disorders after bariatric surgery. Eat Disord. 2013;21:275-82.

11. American Psychiatric Association. Diagnostic and statistical manual of mental disorders. $5^{\text {th }}$ ed. Washington: APA; 2012.

12. Conceição EM, Mitchell JE, Machado PP, Vaz AR, Pinto-Bastos A, Ramalho S, et al. Repetitive eating questionnaire [Rep(eat)-Q]: enlightening the concept of grazing and psychometric properties in a Portuguese sample. Appetite. 2017;117:351-8.

13. Goldschmidt AB, Conceição EM, Thomas JG, Mitchell JE, Raynor HA, Bond DS. Conceptualizing and studying binge and loss of control eating in bariatric surgery patients-time for a paradigm shift? Surg Obes Relat Dis. 2016;12:1622-5.

14. Canetti L, Berry EM, Elizur Y. Psychosocial predictors of weight loss and psychological adjustment following bariatric surgery and a weightloss program: the mediating role of emotional eating. Int $\mathrm{J}$ Eat Disord. 2009;42:109-17.

15. Tatham M, Turner H, Mountford VA, Tritt A, Dyas R, Waller G. Development, psychometric properties and preliminary clinical validation of a brief, session-by-session measure of eating disorder cognitions and behaviors: The ED-15. Int J Eat Disord. 2015;48:1005-15.

16. Pais-Ribeiro JL, Honrado A, Leal I. Contribuição para o estudo da adaptação portuguesa das escalas de ansiedade, depressão e stress (EADS) de 21 itens de Lovibond e Lovibond. Psicol Saúde Doenças. 2004;5:229-39.

17. Lovibond SH, Lovibond PF. Manual for the depression anxiety stress scales. $2^{\text {nd }}$ ed. Sydney: Psychology Foundation; 1995.

18. Whiteside SP, Lynam DR, Miller JD, Reynolds SK. Validation of the UPPS impulsive behavior scale: a four factor model of impulsivity. Eur $J$ Personality. 2005;19:559-74.

19. White MA, Kalarchian MA, Levine MD, Masheb RM, Marcus MD, Grilo CM. Prognostic significance of depressive symptoms on weight loss and psychosocial outcomes following gastric bypass surgery: a prospective 24-month follow-up study. Obes Surg. 2015;25:1909-16.

20. Conceição EM, Mitchell JE, Pinto-Bastos A, Arrojado F, Brandão I, Machado PP. Stability of problematic eating behaviors and weight loss trajectories after bariatric surgery: a longitudinal observational study. Surg Obes Relat Dis. 2017;13:1063-70.

21. Colles SL, Dixon JB, O'Brien PE. Grazing and loss of control related to eating: two high-risk factors following bariatric surgery. Obesity. 2008;16:615-22.

22. Ramalho S, Bastos AP, Silva C, Vaz AR, Brandão I, Machado PP, et al. Excessive skin and sexual function: relationship with psychological variables and weight regain in women after bariatric surgery. Obes Surg. 2015;25:1149-54.

23. Schag K, Mack I, Giel KE, Ölschläger S, Skoda EM, von Feilitzsch M, et al. The impact of impulsivity on weight loss four years after bariatric surgery. Nutrients. 2016;8:721.

24. de Zwaan M, Hilbert A, Swan-Kremeier L, Simonich H, Lancaster K, Howell LM, et al. Comprehensive interview assessment of eating behavior 18-35 months after gastric bypass surgery for morbid obesity. Surg Obes Relat Dis. 2010;6:79-85 